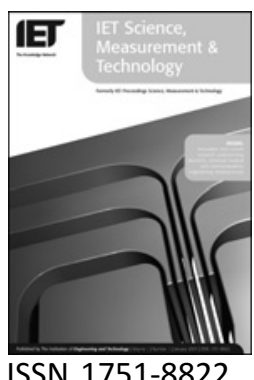

\title{
Smooth boundary topology optimisation applied to an electrostatic actuator
}

\author{
D.-H. Kim ${ }^{1}$ D.A. Lowther ${ }^{2} \quad$ J.K. Sykulski ${ }^{3}$ \\ ${ }^{1}$ School of Electrical Engineering and Computer Science, Kyungpook National University, Daegu 702-701, Korea \\ ${ }^{2}$ Department of Electrical and Computer Engineering, McGill University, Montreal, Quebec H3A 2A7, Canada \\ ${ }^{3}$ School of Electronics and Computer Science, University of Southampton, Southampton SO17 1BJ, UK \\ E-mail: jks@soton.ac.uk
}

\begin{abstract}
Smooth boundary topology optimisation in conjunction with the continuum design sensitivity analysis avoids many of the problems encountered by conventional cell-based systems coupled with material homogenisation or the density method. Shape optimisation becomes part of topology optimisation. The effectiveness of the proposed method is demonstrated through the design of an electrostatic MEMS actuator to generate maximum torque for a predefined maximum size (area).
\end{abstract}

\section{Introduction}

Over the past decade, there has been a growing interest in systems that provide a form of topology optimisation for low-frequency electromagnetic devices. Unlike pure boundary, or shape, optimisation systems - which have received considerable attention in the literature - topology optimisation provides the possibility of developing new geometries to meet design specifications. The approach is closely related to the work on design sensitivity analysis and, indeed, this is a fundamental requirement for efficient topological design. Much of the published research to date has used discrete design sensitivity analysis as the basis for this formulation and the topological variations have been achieved through modifying the material properties in a mesh of rectangular cells $[1,2]$. Sensitivity analysis is used to identify how to change the material properties of each cell in order to achieve the desired performance. However, this approach has two problems. The first is that the results are inherently very 'granular' since the design space is divided into rectangular cells and, thus, the 'smoothness' of the final boundary - or rather the lack of it - is related directly to the original subdivision. This problem may be rectified by simply reducing the size of the cells, thus increasing their number, but this will inevitably lead to a dramatic increase of required computing times. The second problem is that the intermediate states between two real materials are present. Hence penalisation methods are additionally needed in order to retrieve a realistic material, and these are apt to lead to a local optimum solution in practical problems. Recently, an alternate approach has been proposed which uses a continuum version of design sensitivity analysis (CDSA) and, rather than working with rectangular cells, grows areas having smooth boundaries and particular material properties (smooth boundary topology optimisation, SBTO) [3, 4]. It is the intention of this paper to extend the work in SBTO previously reported to a real application of an electrostatic actuator (a MEMS device) and compare the results with those described in an earlier paper using the cell approach [5].

\section{Smooth boundary topology optimisation}

The boundary shape of an object is described in terms of a B-Spline curve; the SBTO modifies this and introduces new and disjoint boundary structures such that a cost function is optimised. The CDSA is used to determine a topological gradient (TG) for boundary and geometry changes. Depending on the TG and a selection criterion $(\mathrm{SC})$ in $[3,4]$, a small region containing either air (if the main region contains material) or material (if the main region contains air) is introduced and can grow and change shape as the optimisation proceeds. Several regions can be created simultaneously and may coalesce into single objects. 


\subsection{Topological gradient}

At any point during optimisation, the device may benefit from the introduction of a small, circular material-filled region $(B(r, d)$ with radius $d$, centred at $r)$ in the design domain $\Omega$. The 'TG', $G(r)$, provides a measure of improvement of the objective function

$$
G(r)=\lim _{d \rightarrow 0} \frac{\Psi_{\text {obj }}(\Omega \backslash B(r, d))-\Psi_{\text {obj }}(\Omega)}{\delta(\Omega)}
$$

where $\Psi_{\text {obj }}$ is the objective function, $\Omega \backslash B(r, d)$ the domain $\Omega$ with the material region $B(r, d)$ and $\delta(\Omega)$ the area difference after and before the small region occurs $[3,4]$. As seen in (1), the definition of the TG is quite different from that of the material sensitivity used commonly in electromagnetic topology optimisation, where the derivative of the objective function with respect to the infinitesimal change of material properties is sought out $[1,2]$.

\subsection{Link with the classical shape optimisation}

The TG and classical shape sensitivity can be shown to be linked and the derivation is given in [3]. In brief, a scalar function, $J$, expressed with $\Psi_{\text {obj }}$ and $d$ is considered

$$
J(d)=\Psi_{\mathrm{obj}}(\Omega \backslash B(r, d))
$$

It is assumed that a small perturbation is introduced in Fig. 1, which keeps the outer boundary $\gamma$ of the domain invariant (design velocity $\boldsymbol{V}=0$ on $\gamma$ ) but increases the radius $d$ of the hole $\left(\boldsymbol{V}=-\boldsymbol{n}\right.$ on $\left.\gamma_{d}\right)$. In this case, the classical shape sensitivity of (2), that is $J^{\prime}$, can be expressed in terms of the electric and adjoint fields based on the shape gradient information [6]

$$
\begin{aligned}
& J^{\prime}(d)=-\int_{\gamma_{d}} L\left(\phi_{1}, \lambda_{1}\right) \mathrm{d} \gamma \\
& L\left(\phi_{1}, \lambda_{1}\right)=\left(\varepsilon_{1}-\varepsilon_{2}\right)\left[\frac{\varepsilon_{1}}{\varepsilon_{2}} p\left(\phi_{1}\right) p\left(\lambda_{1}\right)+q\left(\phi_{1}\right) q\left(\lambda_{1}\right)\right]
\end{aligned}
$$

where $p \equiv \partial / \partial n, q \equiv \partial / \partial t, \phi$ is the electrical potential, $\varepsilon_{1}$ and $\varepsilon_{2}$ refer to either side of the material boundary, whereas $\lambda$ denotes the adjoint variable. Using a local expansion of $\phi$ and $\lambda$ along the circumference of $B(r, d),(3)$ is approximated at the centre of the circular material-filled region as

$$
J^{\prime}(d)=-4 \pi d L\left(\phi_{1}(r), \lambda_{1}(r)\right)+o(d)
$$

Finally, the difference of the objective function after and before inserting the small material-filled region is obtained as given below

$$
\begin{aligned}
J(d)-J(0) & =\int_{0}^{d} J^{\prime}(\rho) d \rho \\
& =-2 \pi d^{2} L\left(\phi_{1}(r), \lambda_{1}(r)\right)+o\left(d^{2}\right)
\end{aligned}
$$

Comparing (5) with (1), the TG gives $G(r)=2 L\left(\phi_{1}(r), \lambda_{1}(r)\right)$, whereas the natural extension of the shape sensitivity is $L\left(\phi_{1}(r)\right.$, $\left.\lambda_{1}(r)\right)$. Hence, the topological and the shape gradients differ by a factor 2 .

\subsection{Introducing a smooth boundary and a material merging process}

The proposed method expresses the boundary of the initial design domain as a B-Spline curve in Fig. $2 a$ and relocates control points, $P_{i}$, of the curve based on the shape sensitivity information at each iterative design stage [7]. Thus, the smooth boundary of the domain can be kept during the overall design process. In the meantime, the topology inside the domain may change by creating a small and circular material-filled region in the domain depending upon the SC. Then, the boundary shape of the inserted material region is defined by a new B-Spline curve as shown in Fig. $2 b$ and is also optimised along with the outer boundary of the domain.

According to the proposed scheme, it may happen that more than two material-filled regions are introduced inside the domain and they intersect each other as the area of each material region grows. In this case, the material
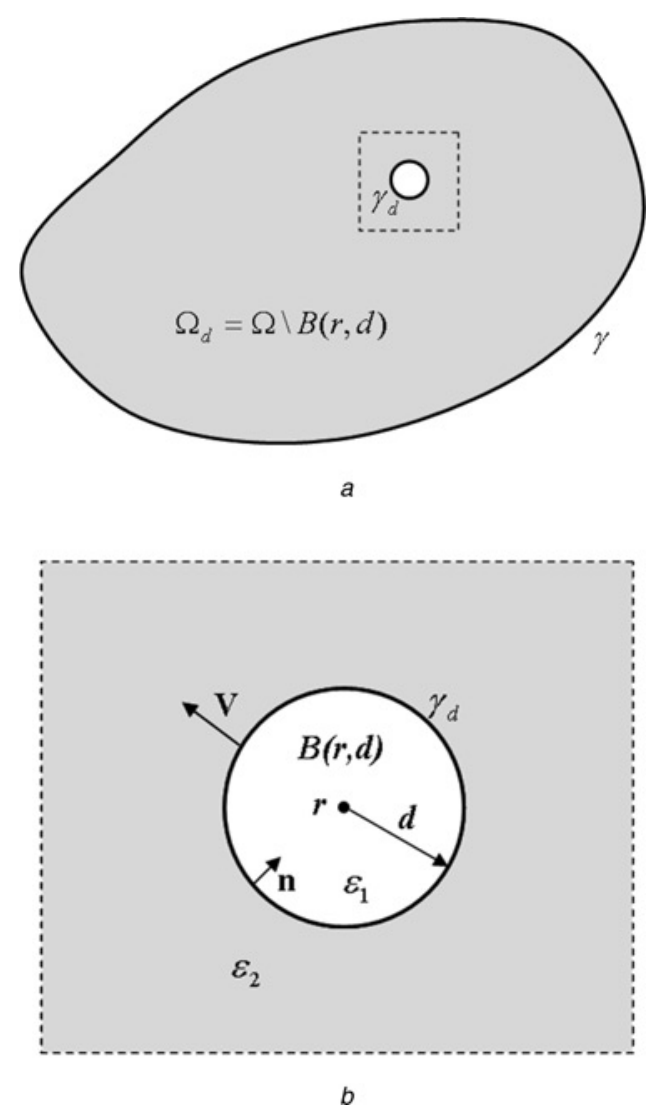

Figure 1 Design domain after cutting a small hole

a Domain $\Omega \backslash B(r, d)$

$b$ Enlarged circular material 


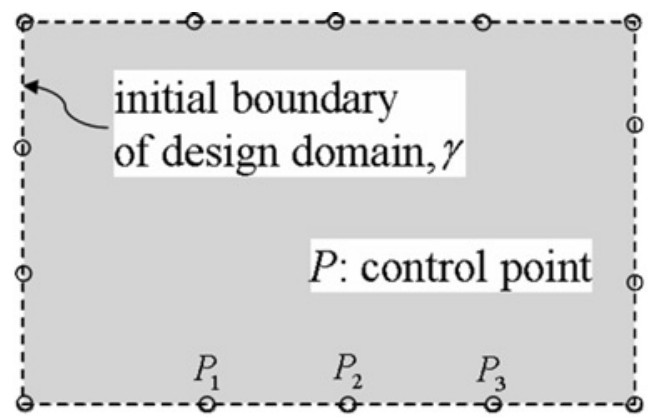

a

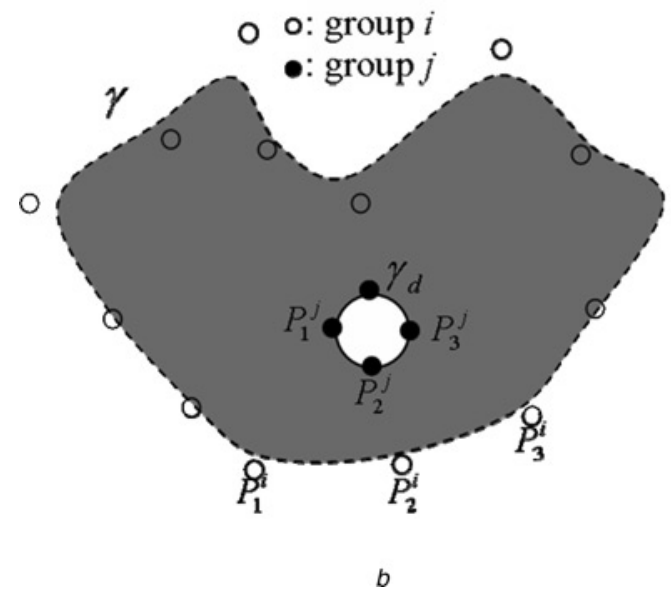

Figure 2 Smooth boundaries of SBTO

a Initial domain

a Topological change

regions should be merged into one in order to avoid geometrical modelling errors that might be produced in commercial EM software packages. Fig. 3 illustrates a material merging process where a new boundary is created by a B-Spline curve with the non-overlapping control points of the two material-filled regions.

\subsection{Selection criterion}

To demonstrate the concept of the SC, it is assumed that the design domain is divided into finite elements. Under the area constraint, first the $\mathrm{SC}$ for the boundary control point, $P_{i}$, of the domain or created material-filled regions is defined as

$$
\mathrm{SC}_{i}^{\text {shape }}=-\frac{\Psi_{\text {obj }}^{\prime}}{\Psi_{\text {const }}^{\prime}}
$$

where $\Psi_{\text {obj }}^{\prime}$ and $\Psi_{\text {const }}^{\prime}$ denote shape sensitivity values of the objective and constraint functions. Similarly, the SC based on the TG values for the $m$ th element inside the discretised domain is also calculated as

$$
\mathrm{SC}_{m}^{\mathrm{topo}}=-\frac{G}{\Psi_{\text {const }}^{\prime}}
$$

Equations (6) and (7) give the sensitivity of the objective function to the change of the constraint function when the

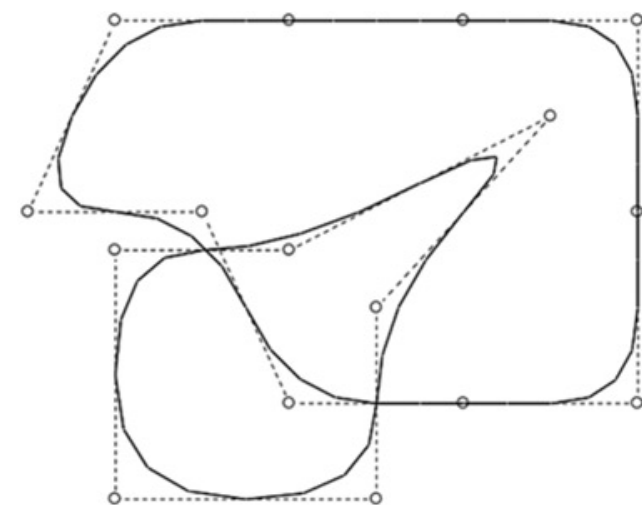

a

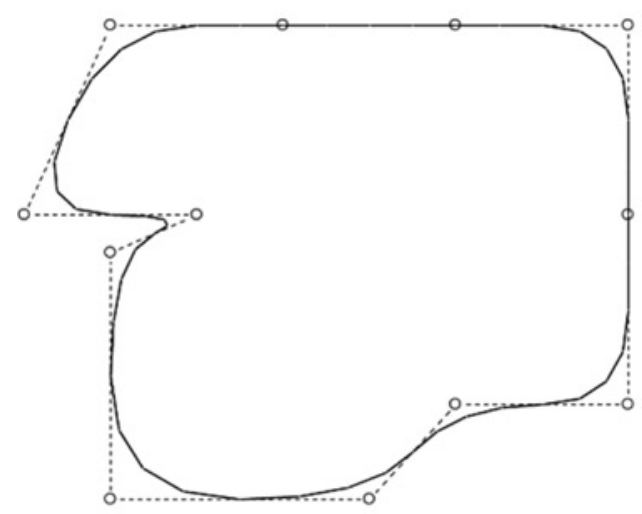

$b$

Figure 3 Illustration of a material merging process

a Before merging process

$b$ After merging process

control point moves or the small and circular material-filled region appears, respectively. Thus, for minimisation problems, the smaller the SC value, the bigger is the contribution to reduce the objective function with respect to the change of the constraint function.

To decide whether the material generation is more beneficial than mere shape optimisation at each iteration, the comparison of the minimum SC values in the two cases is required

$$
\begin{aligned}
\mathrm{SC}_{\min }^{\text {shape }} & \equiv \min \left(\mathrm{SC}_{i}^{\text {shape }}, i=1, \ldots, j\right) \\
\mathrm{SC}_{\min }^{\mathrm{topo}} & \equiv \min \left(\mathrm{SC}_{m}^{\mathrm{topo}}, m=1, \ldots, n\right)
\end{aligned}
$$

where $j$ and $n$ are the total number of control points and finite elements in the domain, respectively. Therefore only if $\mathrm{SC}_{\min }^{\text {topo }}$

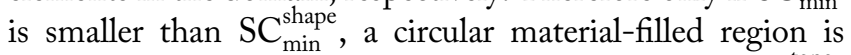
introduced at the element centre corresponding to $\mathrm{SC}_{\min }^{\mathrm{topo}}$. This results in generating a new topology and increasing the number of design variables (control points) at the next iteration. If the area constraint is not considered, the constraint function $\Psi_{\text {const }}^{\prime}$ in (6) and (7) is then set to be a constant value. 
It should be noted that the description above refers to an implementation of the scheme using a finite element field computation software. It must be stressed, however, that the method itself is applicable to any analysis method and does not rely on having any particular type or shape of elements or grids of the numerical technique used or any other method-dependent characteristics of the field solution employed. It has been argued before, but will be repeated here, that the fact that the CDSA approach is not tied up to any particular field modelling formulation constitutes a great strength of the method.

\section{Numerical implementation}

The proposed method basically optimises the boundary shapes of the whole domain, including additionally created material regions, whereas the topology is changed by adding new and circular material-filled regions during design iterations, if necessary. This new methodology was easily combined with a commercial software by utilising an optimisation technique based on CDSA, the prime objective of which is to solve the dual solution system consisting of the primary and adjoint systems $[8,9]$.

The iterative design process for SBTO, shown in Fig. 4, involves the following steps.

Step 1: Specify the initial design domain with a B-Spline curve and its control points.

Step 2: Set design variables and impose side/area constraints.

Step 3: Analyse the primary and adjoint systems.

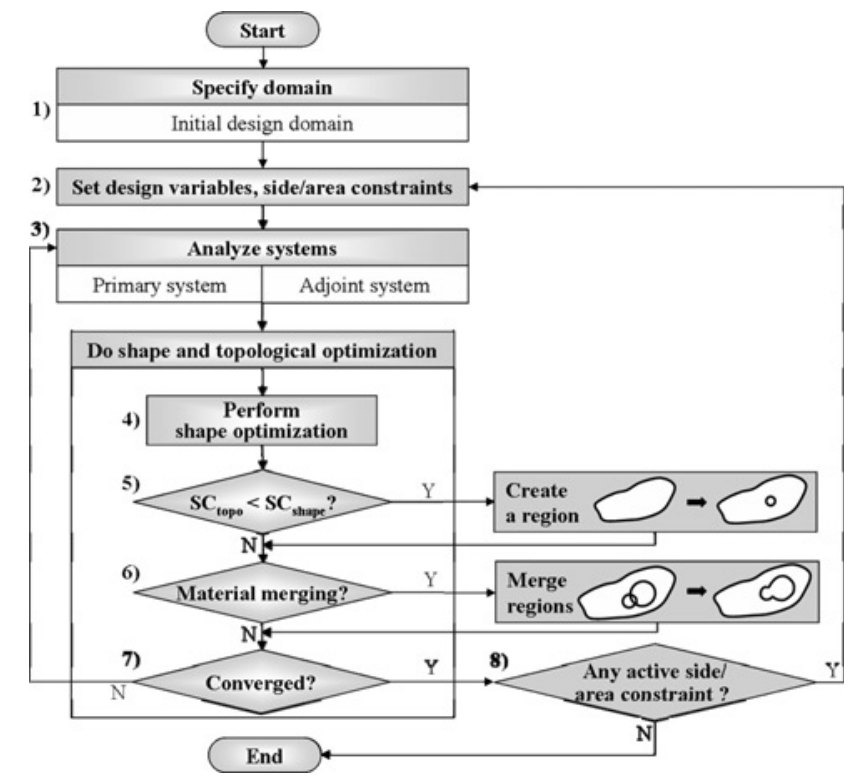

Figure 4 Convergence of the objective function against iterations
Step 4: Compute the shape and the TG values and then execute shape optimisation.

Step 5: Compute SC values and insert a small and circular material-filled region if necessary.

Step 6: Merge two material regions into one region if they intersect.

Step 7: Check convergence and go to step 3 if unsatisfactory.

Step 8: Check side/area constraints and go to step 2 if unsatisfactory.

\section{$4 \quad$ Results}

An electrostatic actuator [5] with 16 electrodes is shown in Fig. 5 where the radius of the electrode is $100 \mu \mathrm{m}$ and the width of the air gap is $5 \mu \mathrm{m}$. The actuator is driven by activating each anode-cathode pair (the electrodes).

The design goal is to create a dielectric rotor producing as large a torque as possible. The outline of the maximum size of the rotor is restricted to a $95 \mu \mathrm{m}$ radius, as depicted in Fig. 5, but this architecture would, of course, generate no torque if the design domain were to be fully occupied by dielectric. The design goal is achieved by minimising the following objective function under the area constraint condition given

$$
\begin{aligned}
\Psi_{\text {obj }} & =\left(W_{A}-W_{A o}\right)^{2}+\left(W_{B}-W_{B o}\right)^{2} \\
\Psi_{\text {const }} & =\left(A_{k}-A_{k o}\right)^{2} \leq \delta
\end{aligned}
$$

where positions $A$ and $B$ activating each electrode pair are $22.5^{\circ}$ apart, $W_{A \mathrm{o}}$ is the energy when the initial design space is fully occupied by air, $W_{B o}$ refers to the energy when the area is completely filled with dielectric, $A_{k}$ is the area occupied by dielectric material, $A_{k o}$ is the target area and $\delta$ denotes a constant value corresponding to the area difference of $45 \%$. A side constraint is additionally imposed on the B-Spline curve forming the boundaries of the

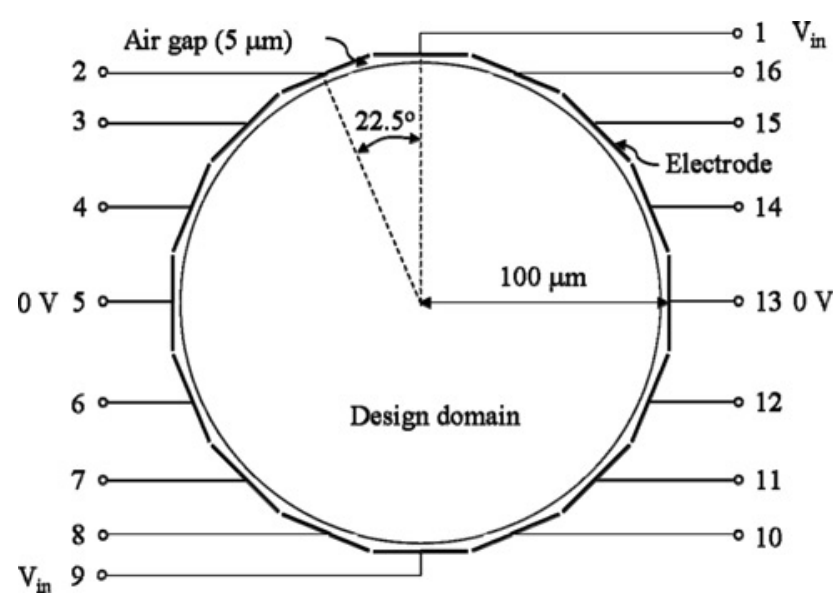

Figure 5 Actuator layout and design domain 
dielectric regions not to exceed the maximum radius of $95 \mu \mathrm{m}$.

The optimisation starts with an initial shape and topology where the design domain has a circular dielectric region with a radius of $20 \mu \mathrm{m}$ and a relative permittivity value of 10 (Fig. 6a). To compute the shape and TG values, there is no need to analyse the adjoint system in this problem because the objective function is expressed in terms of the system stored energy $[10,11]$. At each iteration, the system decides whether a new material region should be created or whether just a change to the boundary shape will be sufficient. This information is derived from the comparison of the SC values shown in Fig. 4. The evolution of the rotor is shown in Fig. 6 as the design iterations proceed. The boundary shape of the dielectric varies and also new

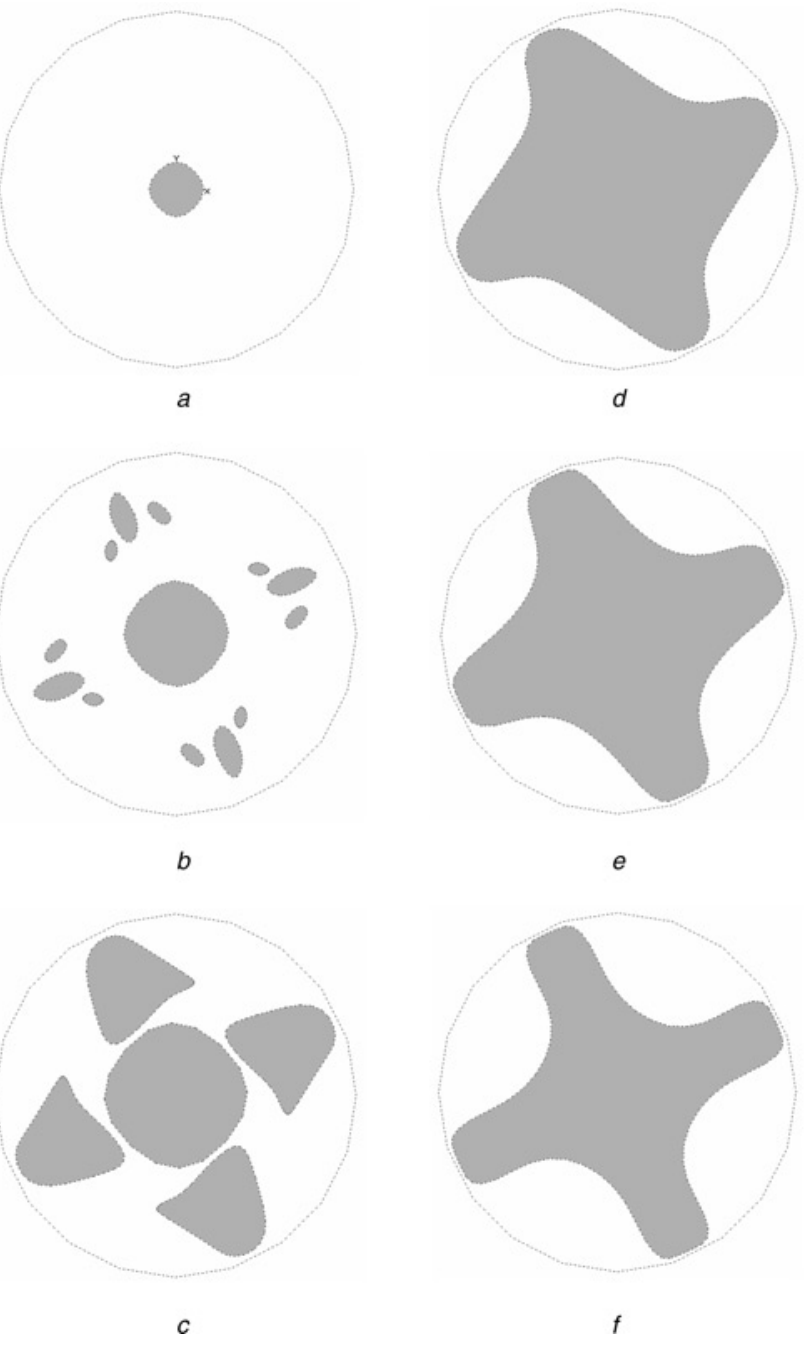

Figure 6 Insertion and evolution of material regions while minimising the objective function
a 0 iteration
$b 5$ iterations
c 8 iterations
d 10 iterations
e 15 iterations
$f 29$ iterations

dielectric regions are created during five iterations as shown Fig. $6 b$. The adjacent dielectric regions are merged into one region in Figs. $6 c$ and $6 d$ when they intersect each other. After 29 iterations, the final shape and topology satisfying the area constraint are obtained (Fig. 6f).

Fig. 7 compares the final optimised shapes of the rotor, achieved using SBTO and the density method [5]. The
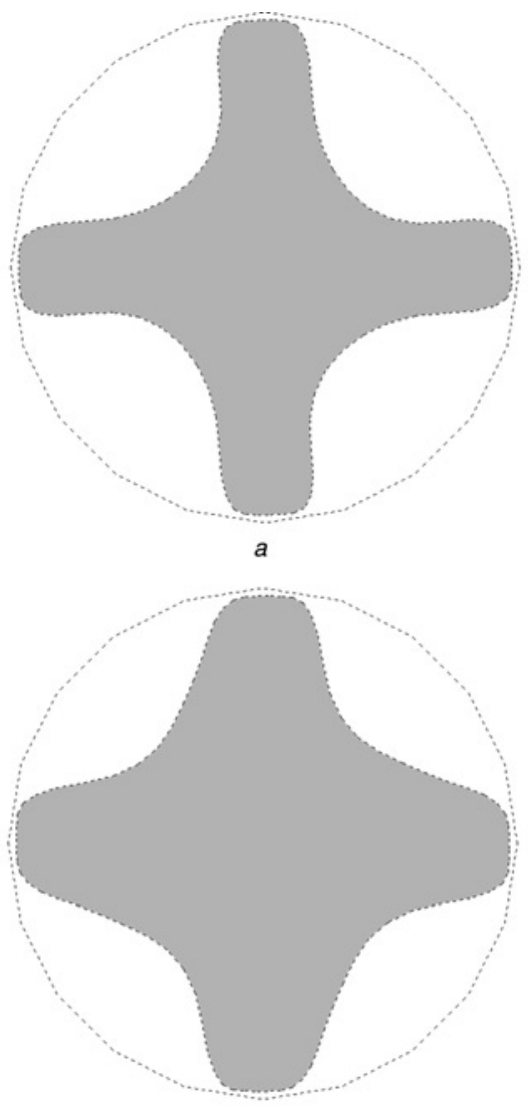

b

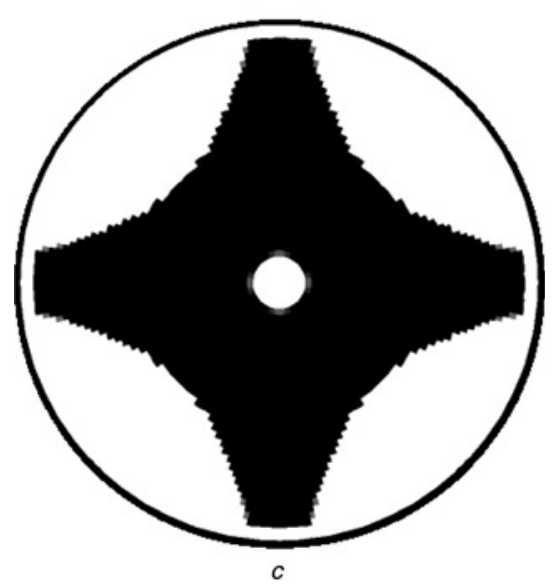

Figure 7 Comparison of optimised rotors between two methods

a After 29 iterations using SBTO with dielectric area constraint $b$ After 17 iterations using SBTO without dielectric area constraint c After 100 iterations using the density method of [5] without dielectric area constraint 


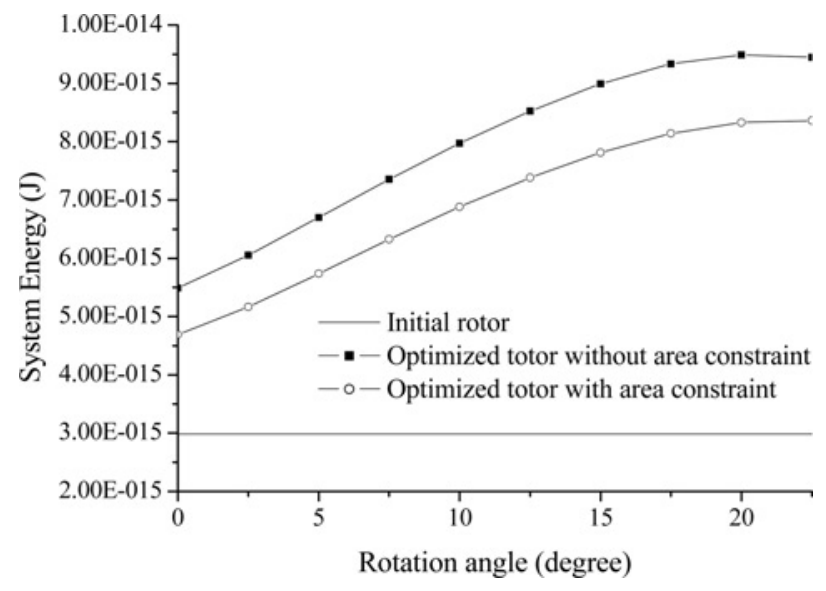

Figure 8 Energy profile before and after optimisation

shapes appear to be quite similar but the density method produces the 'staircase' effect on the boundary which the SBTO does not. Moreover, it was reported in [5] that the final topology depends strongly on the element size used and the initial material values imposed, which lead to possible local minima traps (different topologies). However, this defect of the density method has not been identified as a problem in SBTO. In addition, it was revealed that fewer iterations, typically less than 30 , were needed to achieve convergence in SBTO when compared with the density method which required more than 100 iterations for an optimum solution.

The comparisons of the energy variations and the torque profiles of the optimised designs is presented in Figs. 8 and 9. It is worth highlighting that the maximum torque produced by the proposed method is more than 20\% larger than that achieved in [5].

\section{Robust design and topology optimisation}

In this paper, the method described is intended to optimise the topology of a device, rather than just the shape. This

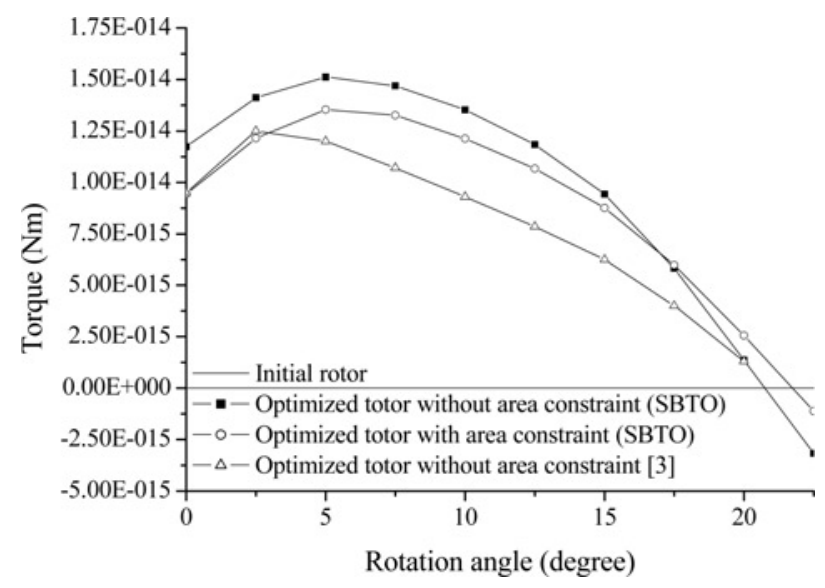

Figure 9 Torque profile before and after optimisation method has implications in the area of robust design and it is, perhaps, worth commenting on this briefly. Robust design is usually associated with creating a design which performs to a required specification within the accepted bounds of manufacturing errors, that is, variations in material properties and component dimensions. It could be argued that the method proposed in this paper partly addresses this issue since the topology optimisation is implemented based on constraints on the physical dimensions and the properties of the provided material. The TG constitutes, by itself, a measure of robustness of the solution; if the gradient is too large, then the material properties in the region considered should be changed. By using the value of the TG, the sensitivity of the design solution to variations in both a material property and a physical dimension can be determined. Thresholding this value can then provide some information on the robustness of the design.

\section{Conclusions}

From the results, it has been revealed that the SBTO shows fast convergence with respect to the objective function given and also is relatively free from possible local minima traps. Moreover, the method avoids the 'staircase' effects that are encountered in the conventional cell-based systems coupled with material homogenisation or the density method. The topology finally generated compares very favourably with that developed in [5], while offering improved performance. The relationship between robustness and topological sensitivity is intended to be further investigated in future work.

\section{Acknowledgments}

This work was supported by the Korea Science and Engineering Foundation (KOSEF) grant funded by the Korean government (MEST) (No. R01-2008-000-10522-0).

\section{References}

[1] DYCK D.N., LOWTHER D.A., FREemAn E.M.: 'A method of computing the sensitivity of electromagnetic quantities to changes in material and sources', IEEE Trans. Magn., 1994, 30, pp. $3415-3418$

[2] KIM D., SYKULSKI J.K., LOWTHER D.A.: 'A novel scheme for material updating in source distribution optimisation of magnetic devices using sensitivity analysis', IEEE Trans. Magn., 2005, 41, pp. 1752-1755

[3] Cea J., garreau S., guillaume P., masmoudi M.: 'The shape and topological optimisations connection', Comput. Methods Appl. Mech. Eng., 2000, 118, pp. 713-726

[4] KIM D., LEE S., KWANK M., KIM H., LOWTHER D.A.: 'Smooth boundary topology optimisation for electrostatic problems 
through the combination of shape and topological design sensitivities'. Proc. Compumag, Aachen, Germany, 2007, PC3-10

[5] BYUN J., PARK I., HAHN S.: 'Topology optimisation of electrostatic actuator using design sensitivity', IEEE Trans. Magn., 2002, 38, pp. 1053-1056

[6] KIM D., PARK I., SHIN M., SYKULSKI J.K.: 'Generalized continuum sensitivity formula for optimum design of electrode and dielectric contours', IEEE Trans. Magn., 2003, 39, pp. 1281-1284

[7] KIM C., LEE H., PARK I.: 'B-spline parameterization of finite element models for optimal design of electromagnetic devices', IEEE Trans. Magn., 1999, 35, pp. 3763-3765
[8] PARK I., COULOMB J.L., HAHN S.: 'Implementation of continuum sensitivity analysis with existing finite element code', IEEE Trans. Magn., 1993, 29, pp. 1787-1790

[9] Infolytica Corporation MagNet 6 User's Guide, 2005

[10] KIM D., LEE S., PARK I., LEE J.: 'Derivation of a general sensitivity formula for shape optimisation of 2-D magnetostatic systems by continuum approach', IEEE Trans. Magn., 2002, 38, pp. 1125-1128

[11] KIM D., SHIP K., SYKULSKI J.K.: 'Applying continuum design sensitivity analysis combined with standard EM software to shape optimisation in magnetostatic problems', IEEE Trans. Magn., 2004, 40, pp. 1156-1159 